Acta Crystallographica Section A

Foundations of

Crystallography

ISSN 0108-7673

Received 17 October 2006

Accepted 11 January 2007

\section{Comment on On the calculation of the electrostatic potential, electric field and electric field gradient from the aspherical pseudoatom model by Volkov, King, Coppens \& Farrugia (2006)}

\author{
Mark A. Spackman \\ Chemistry - M313, School of Biomedical, Biomolecular \& Chemical Sciences, University of Western Australia, \\ Crawley, WA 6009, Australia. Correspondence e-mail: mas@cyllene.uwa.edu.au
}

Printed in Singapore - all rights reserved

incorporating vibrational (or thermal) averaging of deformation electron densities closely approximate static results in regions far from the nuclei.

A number of papers that emerged from Stewart's group in the early 1980s outlined Fourier summation, direct space and combined strategies for the computation of the ESP, EF and EFG, with examples drawn from pseudoatom multipole fits to experimental data for imidazole and 9-methyladenine measured by Craven's group at the University of Pittsburgh (Spackman \& Stewart, 1981; Stewart, 1982; Spackman \& Stewart, 1984). Later applications of this kind included a combined Fourier/direct-space approach to mapping the total ESP in sodium zeolite A (Spackman \& Weber, 1988), and a detailed presentation of ESP maps for molecules and molecular clusters of urea, imidazole, 9-methyladenine and benzene (Stewart, 1991). All of these results were based on algorithms developed by Stewart that were unfortunately not published at the time. They were incorporated into early versions of $V A L R A Y$, and the 1983 version of $V A L R A Y$ implemented the entire suite of Fourier, direct-space and combined approaches to the computation of the ESP, EF and EFG for isolated molecules, clusters or the crystal. Stewart's expressions for the direct-space computation of the ESP from the parameters of the pseudoatom model were also incorporated into MOLPOT, part of the series of programs known as the 'POP procedure' (He, 1984; Craven et al., 1987; Craven, 1988). MOLPOT in turn formed the basis for ELECTROS (Ghermani et al., 1992), and Ghermani et al. (1993) have published an independent derivation of the relevant expressions for the ESP.

Su \& Coppens (1992) published the first complete derivation and expressions for computing not only the ESP but also the EF and EFG from pseudoatom model parameters, including penetration (peripheral) contributions, based on a generalization of the Fourier convolution approach used by Epstein \& Swanton (1982). Their expressions were exceedingly complex, and according to VKCF involved 'the evaluation of fairly complicated one-electron twocentre integrals', and further comments in the recent paper suggest that their incorporation into the $X D$ suite was never fully satisfactory. The VKCF paper presents a careful review of the derivation of the expressions published by Ghermani et al. (1993) and, instead of the Green function approach used by Ghermani et al., VKCF expand $\left|\mathbf{r}-\mathbf{r}^{\prime}\right|^{-1}$ directly in real spherical harmonics, along the lines of previous work (Bentley, 1981; te Velde, 1990; De Bondt et al., 1993; te Velde et al., 2001).

Stewart's detailed derivation for the ESP was published in conjunction with Craven in a study on $\gamma$-aminobutyric acid (Stewart \& Craven, 1993). It presents Stewart's beautiful and elegant derivation of the same expressions published by others, including the closed 
forms of equations (22) and (23) in VKCF, and based on the Fourier convolution approach. The key difference between this and other derivations is that Stewart's makes use of his intimate knowledge of Bessel functions and their inter-relationships. This is more than a mathematical curiosity; it enabled relatively straightforward extension of the same approach to the derivation of expressions for the EF and EFG, yielding separate expressions for all three components of the EF, and for all five components of the traceless EFG tensor. As noted above, these latter derivations and expressions were never published in the peer-reviewed literature, but they were incorporated in Appendix C of the VALRAY manual in 2000 (Stewart et al., 2000). As that manual has not been widely circulated, in the interests of wider dissemination of those important results, that Appendix is provided in PDF format as supplementary material accompanying this communication. ${ }^{\mathbf{1}}$

This Letter concludes with some remarks pertaining to the results and discussion in $\S 4$ of the paper by VKCF. The first concerns the EF, and in particular the map of EF projected onto the plane of the formamide molecule (Fig. 1 of VKCF), based on a pseudoatom modelling of theoretical structure factors. The contribution of the 'central' molecule has been omitted from the map and, as VKCF emphasize, the resulting map very nicely demonstrates how the 'central' molecule experiences an EF due to surrounding molecules that is closely parallel to its own dipole moment vector. This is an important result, and the strategy used to obtain it, namely subtracting the contribution from a particular molecule (or atom or ion), deserves to be used more widely. In light of the comments above, it is worth noting that the map presented by VKCF is based on a direct-space summation of contributions from only the eight nearest neighbours in the crystal. The computation of the EF in the crystal due to point dipoles is well known to require lattice summation techniques to achieve convergence (Cummins et al., 1976), and that is one reason why Stewart advocated combined Fourier/direct-space approaches to computing some properties, and these combined approaches are entirely analogous to the Ewald approach used to achieve rapid convergence of lattice sums. Thus, computation of the ESP and EF in the crystal are best achieved with an approach involving both summation over appropriate Fourier coefficients (e.g. those due to the deformation density) as well as a direct-space calculation (e.g. over spherical atoms, including nuclei).

Regarding the EFG, VKCF write (p. 401): 'Several methods for the calculation of the ESP/EFG were proposed by Brown \& Spackman (1994)', and 'While giving more or less reasonable results, these methods are either too computationally demanding or have convergence problems with Fourier sums.' These comments rather miss the point of the calculations presented in that paper. We noted in that paper that, at that time, the direct-space EFG code in VALRAY had never been tested fully, and the purpose of the various computational routes to the EFG was simply to validate that code in VALRAY (not the algorithms). Thus, we compared results and convergence behaviour of the direct-space EFG with: (i) a combined Fourier/directspace approach as described above for the EF; and (ii) numerical second differentiation of both the direct-space and combined Fourier/ direct-space approaches to the ESP. It was never our intention that any approach other than the direct-space calculation be used to compute the EFG from the pseudoatom model; we did locate minor bugs and proceeded to validate the corrected code.

\footnotetext{
${ }^{1}$ Supplementary material for this paper is available from the IUCr electronic archives (Reference: SH5054). Services for accessing this material are described at the back of the journal.
}

Also on p. 401, VKCF state that 'the method of Su \& Coppens only allows calculation of the traceless EFG tensor at the nuclear positions'. Equation (1) of their paper, Poisson's equation, relates the Laplacian of the ESP to the electron density, and hence any code that computes the electron density and the traceless EFG can also provide the complete EFG tensor, although only the traceless EFG tensor is relevant in the computation of quantities such as nuclear quadrupole coupling constants measured in NMR or NQR experiments (Cohen \& Reif, 1957). In their comparison between EFG results derived from pseudoatom modelling of theoretical structure factors for formamide (labelled $\mathrm{XD} / \mathrm{PBE} / 6-31 \mathrm{G}^{* *}$ in their tables) and those directly from the $a b$ initio wavefunction (labelled PBE/6-31G**), VKCF use the whole EFG tensor (i.e. not the traceless tensor), which appears to obscure any agreement between the two. Although VKCF (p. 405) conclude that agreement between the two is 'very good, taking into account the differences between Gaussian- and Slater-type functions', this reflects the systematic differences between electron densities at the nuclei. Converting their results (reported in their Tables 4 and 5) to traceless form reveals that for $\mathrm{H}$ atoms their $\mathrm{XD} /$ $\mathrm{PBE} / 6-31 \mathrm{G}^{* *}$ results are in almost perfect agreement with those obtained directly from the wavefunction, and that (to only the single significant figure available) a similar result holds for the $\mathrm{C}$ atom, but agreement gets progressively worse for $\mathrm{N}$ and $\mathrm{O}$, atoms with more contracted electron densities. These conclusions are in excellent agreement with our own detailed model studies on a number of molecular crystals (Spackman \& Byrom, 1996; Spackman et al., 1999), with our earlier work on benzene and corundum (Brown \& Spackman, 1994), and they clearly echo conclusions by Epstein et al. (1977), based on generalized scattering factor (g.s.f.) expansions for diatomic molecules.

A final comment concerns the use of Sternheimer corrections by VKCF in their computation of the EFG tensor at the Fe nucleus based on multipole modelling of X-ray data for iron pentacarbonyl. The use of Sternheimer corrections was never discussed by Stewart, nor in our own work, yet appears in analyses by Coppens and coworkers [for a detailed discussion and summary of many results, see Section 10.3 of Coppens's monograph (Coppens, 1997), and Section VII.C of the review article by Koritsanszky \& Coppens (2001)], and has been used without question in recent analyses (Dahaoui et al., 2001). However, the use of Sternheimer corrections deserves to be questioned, as they were introduced to describe so-called 'core polarization' at a time when EFGs in solids were estimated using point charges on atomic sites. VKCF incorporate both a shielding factor, $R^{\text {core }}$, and an antishielding factor, $\gamma_{\infty}$, resulting in an expression for the EFG tensor components for the Fe nucleus, $E_{\alpha \beta}(\mathbf{r})=0.927 E_{\alpha \beta}^{\text {central }}(\mathbf{r})+9.933 E_{\alpha \beta}^{\text {peripheral }}(\mathbf{r})$. Thus, the contribution from the nucleus-centred quadrupole functions is reduced by $\sim 7 \%$, and the (generally much smaller) peripheral (penetration) contribution is enhanced by an order of magnitude by including these correction factors. Tsirelson \& Ozerov (1996) have argued that the antishielding correction should only be included when the peripheral term is approximated by a point-charge model, and this makes a great deal of sense. It is also arguable that even the shielding contribution should be included. Coppens (1997) argued that its use takes into account the fact that the pseudoatom model uses a frozen core approximation, and thus cannot describe core polarization effects. However, Schwarz, Blaha and co-workers have performed accurate all-electron theoretical calculations of EFGs in a variety of complex solids using a full-potential linearized augmented plane-wave method (FP LAPW) and make no use of any Sternheimer corrections (Blaha et al., 1985; Schwarz \& Blaha, 1992; Dufek et al., 1995; Blaha et al., 2000; Schwarz \& Blaha, 2003). Most importantly, their calculations 
provide a breakdown of the EFG tensors into contributions from semi-core (SC) and valence states. For a series of $\mathrm{Al}_{2} \mathrm{SiO}_{5}$ polymorphs (Iglesias et al., 2001), they conclude that the 'main contribution to the EFG comes from the distortion from spherical symmetry of the respective valence $p$ electrons', although for $\mathrm{Al}$ and $\mathrm{Si}$ important contributions come from the low-lying semi-core states on $\mathrm{Al}(2 p)$ and $\mathrm{O}(2 s)$. Similarly, results for a series of Fe-containing solids indicate that 'usually the Fe $3 p$ SC state contribution is less than $10 \%$ of the valence part' (Dufek et al., 1995). This is not intended to suggest that core polarization is negligible, but it seems likely that more important factors currently hamper determination of accurate EFG tensors for heavy atoms from X-ray diffraction data (e.g. a complete and accurate description of the thermal motion of the nucleus, limited flexibility of radial functions in the pseudoatom model, and inherent limits on the resolution of the X-ray diffraction data), and the use of Sternheimer shielding factors is likely to obscure any attempts at improvement in these areas.

It is worth reiterating that the new expressions and algorithms presented by VKCF and especially their incorporation into $X D$ - now used in more than three-quarters of published experimental chargedensity studies - represents an important step towards the routine determination and presentation of ESP, EF and EFG results based on modern charge-density studies. In particular, it would be a suitably fitting outcome of the incorporation of the new expressions by VKCF into $X D$ if attention could be focused on the EFG tensor once again, with the aim of seeking quantitative agreement with NQR results where possible, as advocated by Stewart (1977) nearly 30 years ago.

I am most indebted to Bryan Craven and Joseph Ho (Xiao-Min $\mathrm{He}$ ) for their comments regarding the incorporation of Stewart's algorithms into the POP series of programs at the University of Pittsburgh. Thanks are also due to Henning Sørensen, who kindly confirmed for me the contents of various versions of the VALRAY manual.

\section{References}

Bentley, J. (1981). Chemical Applications of Atomic and Molecular Electrostatic Potentials, edited by P. Politzer \& D. G. Truhlar, pp. 63-84. New York: Plenum.

Bertaut, E. F. (1978). J. Phys. Chem. Solids, 39, 97-102.

Blaha, P., Schwarz, K., Faber, W. \& Luitz, J. (2000). Hyperfine Inter. 126, 389-395.

Blaha, P., Schwarz, K. \& Herzig, P. (1985). Phys. Rev. Lett. 54, 1192-1195.

Brown, A. S. \& Spackman, M. A. (1994). Mol. Phys. 83, 551-566.

Cohen, M. H. \& Reif, F. (1957). Solid State Phys. Adv. Res. Applic. 5, 321-438.

Coppens, P. (1997). X-ray Charge Densities and Chemical Bonding. New York: Oxford University Press.

Craven, B. M. (1988). Crystallographic Computing 4. Techniques and New Technologies, edited by N. W. Isaacs \& M. R. Taylor, pp. 211-220. Oxford University Press.
Craven, B. M., Weber, H.-P. \& He, X. (1987). Report TR-87-2, University of Pittsburgh, PA, USA.

Cummins, P. G., Dunmur, D. A., Munn, R. W. \& Newham, R. J. (1976). Acta Cryst. A32, 847-853.

Dahaoui, S., Ghermani, N. E., Ghose, S. \& Howard, J. A. K. (2001). Am. Mineral. 86, 159-164.

De Bondt, H. L., Blaton, N. M., Peeters, O. M. \& De Ranter, C. J. (1993). Z. Kristallogr. 208, 167-174.

Dufek, P., Blaha, P. \& Schwarz, K. (1995). Phys. Rev. Lett. 75, 3545-3548.

Epstein, J., Bentley, J. \& Stewart, R. F. (1977). J. Chem. Phys. 66, 5564-5567.

Epstein, J. \& Swanton, D. J. (1982). J. Chem. Phys. 77, 1048-1060.

Ghermani, N., Lecomte, C. \& Bouhmaida, N. (1993). Z. Naturforsch. Teil A, 48, 91-98.

Ghermani, N. E., Bouhmaida, N. \& Lecomte, C. (1992). ELECTROS: Computer Program to Calculate Electroststic Properties from High Resolution X-ray Diffraction. Internal Report URA CNRS 809, Université de Nancy I, France.

He, X. M. (1984). PhD thesis, University of Pittsburgh, PA, USA.

Iglesias, M., Schwarz, K., Blaha, P. \& Baldomir, D. (2001). Phys. Chem. Miner. 28, 67-75.

Koritsanszky, T. \& Coppens, P. (2001). Chem. Rev. 101, 1583-1627.

Koritsanszky, T., Howard, S. T., Macchi, P., Gatti, C., Farrugia, L. J., Mallinson, P. R., Volkov, A., Su, Z., Richter, T. \& Hansen, N. K. (2003). XD - A Computer Program Package for Multipole Refinement and Analysis of Charge Densities from Diffraction Data, 4.10 ed. Middle Tennessee State University, TN, USA; University of Milano, Italy; University of Glasgow, UK.

Schwarz, K. \& Blaha, P. (1992). Z. Naturforsch. Teil A, 47, 197-202.

Schwarz, K. \& Blaha, P. (2003). Comput. Mater. Sci. 28, 259-273.

Schwarzenbach, D. \& Thong, N. (1979). Acta Cryst. A35, 652-658.

Spackman, M. A. \& Byrom, P. G. (1996). Acta Cryst. B52, 1023-1035.

Spackman, M. A., Byrom, P. G., Alfredsson, M. \& Hermansson, K. (1999). Acta Cryst. A55, 30-47.

Spackman, M. A. \& Stewart, R. F. (1981). Chemical Applications of Atomic and Molecular Electrostatic Potentials, edited by P. Politzer \& D. G. Truhlar, pp. 407-425. New York: Plenum.

Spackman, M. A. \& Stewart, R. F. (1984). Methods and Applications in Crystallographic Computing, edited by S. R. Hall \& T. Ashida, pp. 302-320. Oxford: Clarendon Press.

Spackman, M. A. \& Weber, H.-P. (1988). J. Phys. Chem. 92, 794-796.

Stewart, R. F. (1977). Chem. Phys. Lett. 49, 281-284.

Stewart, R. F. (1979). Chem. Phys. Lett. 65, 335-342.

Stewart, R. F. (1982). God. Jugosl. Cent. Kristalogr 17, 1-24.

Stewart, R. F. (1991). The Application of Charge Density Research to Chemistry and Drug Design, edited by G. A. Jeffrey \& J. F. Piniella, pp. 63-102. New York: Plenum Press.

Stewart, R. F. \& Craven, B. M. (1993). Biophys. J. 65, 998-1005.

Stewart, R. F., Spackman, M. A. \& Flensburg, C. (2000). VALRAY - User's Manual, 2.1 ed. Carnegie Mellon University, Pittsburgh, PA, USA, and University of Copenhagen, Denmark.

Su, Z. \& Coppens, P. (1992). Acta Cryst. A48, 188-197.

Tsirelson, V. \& Ozerov, R. P. (1996). Electron Density and Bonding in Crystals. London: IOP Publishing.

Velde, G. te (1990). PhD thesis, Vrije Universiteit, Amsterdam, The Netherlands.

Velde, G. te, Bickelhaupt, F. M., Baerends, E. J., Fonseca Guerra, C., van Gisbergen, S. J. A., Snijders, J. G. \& Ziegler, T. (2001). J. Comput. Chem. 22, 931-967.

Volkov, A., King, H. F., Coppens, P. \& Farrugia, L. J. (2006). Acta Cryst. A62, 400-408. 
Acta Crystallographica Section A

Foundations of Crystallography

ISSN 0108-7673

Received 3 January 2007

Accepted 11 January 2007

\section{Response to Spackman's comment on On the calculation of the electrostatic potential, electric field and electric field gradient from the aspherical pseudoatom model}

\author{
Anatoliy Volkov* and Philip Coppens*
}

Department of Chemistry, University at Buffalo, State University of New York, Buffalo, NY 14260-3000, USA.

Correspondence e-mail: volkov@chem.buffalo.edu,coppens@buffalo.edu
(C) 2007 International Union of Crystallography Printed in Singapore - all rights reserved
Table 1

Root-mean-squared differences in electric field components $E_{x}$ and $E_{y}\left(\mathrm{e} \AA^{-2}\right)$ in the plane of the map between exact and EPMM calculations .

(R.m.s. values of $E_{x}$ and $E_{y}$ are $\sim 5.4$ and $\sim 6.5 \mathrm{e} \AA^{-2}$, respectively.)

\begin{tabular}{lllll}
\hline & $\begin{array}{l}8 \text { neighboring } \\
\text { molecules }\end{array}$ & $\begin{array}{l}\text { All atoms with } \\
-1<x, y, z<2\end{array}$ & $\begin{array}{l}\text { All atoms with } \\
-2<x, y, z<3\end{array}$ & $\begin{array}{l}\text { All atoms with } \\
-3<x, y, z<4\end{array}$ \\
\hline R.m.s. $\left(E_{x}\right)$ & $2 \times 10^{-6}$ & $1 \times 10^{-5}$ & $1 \times 10^{-5}$ & $1 \times 10^{-5}$ \\
R.m.s. $\left(E_{y}\right)$ & $2 \times 10^{-6}$ & $7 \times 10^{-6}$ & $1 \times 10^{-5}$ & $1 \times 10^{-5}$ \\
\hline
\end{tabular}

Table 2

Number of atoms and elapsed time (s) for each of the calculations (calculations were performed using Athlon64 3400+2.2 GHz processor with 1MB of L2 cache).

\begin{tabular}{|c|c|c|c|}
\hline \multirow[b]{2}{*}{ Calculation } & \multicolumn{2}{|c|}{ Elapsed CPU time (s) } & \multirow{2}{*}{$\begin{array}{l}\text { Number of atoms } \\
\text { used in the } \\
\text { calculation }\end{array}$} \\
\hline & Exact formulae & EPMM & \\
\hline 8 neighboring molecules & 5.2 & 4.3 & 48 \\
\hline All atoms with $-1<x, y, z<2$ & 54.5 & 13.6 & 642 \\
\hline All atoms with $-2<x, y, z<3$ & 254.5 & 40.7 & 2994 \\
\hline All atoms with $-3<x, y, z<4$ & 713.8 & 100.8 & 8226 \\
\hline All atoms with $-4<x, y, z<5$ & - & 216.5 & 17490 \\
\hline
\end{tabular}

Table 3

Root-mean-squared differences in electric field components $E_{x}$ and $E_{y}\left(\mathrm{e} \AA^{-2}\right)$ in the plane of the map relative to the values from calculation that included all atoms with $-4<x, y, z<5$ (all from EPMM calculations).

(R.m.s. values of $E_{x}$ and $E_{y}$ are $\sim 5.4$ and $\sim 6.5 \mathrm{e} \AA^{-2}$, respectively.)

\begin{tabular}{lllll}
\hline & $\begin{array}{l}8 \text { neighboring } \\
\text { molecules }\end{array}$ & $\begin{array}{l}\text { All atoms with } \\
-1<x, y, z<2\end{array}$ & $\begin{array}{l}\text { All atoms with } \\
-2<x, y, z<3\end{array}$ & $\begin{array}{l}\text { All atoms with } \\
-3<x, y, z<4\end{array}$ \\
\hline R.m.s. $\left(E_{x}\right)$ & 0.02 & 0.006 & 0.0007 & 0.00020 \\
R.m.s. $\left(E_{y}\right)$ & 0.03 & 0.004 & 0.0004 & 0.00009 \\
\hline
\end{tabular}

well aware of the existence of the Ewald summation techniques which we used previously in the calculation of the lattice energy of ionic ( $\mathrm{Su}$ \& Coppens, 1995) and molecular (Abramov, Volkov, Wu \& Coppens, 2000a,b; Abramov, Volkov \& Coppens, 2000) crystals. However, the direct-space summation was found to be advantageous in terms of both speed and ease of implementation when combined with the new EPMM method (Volkov et al., 2007).

MS states that we misunderstand the purpose of the methods described by Brown \& Spackman (1994) as several algorithms published in this paper were used only for debugging of the $V A L R A Y$ code and should not be considered of practical importance. 
Table 4

Components of the traceless EFG tensor at the nuclear positions in formamide (atomic units) from different methods.

\begin{tabular}{|c|c|c|c|c|c|c|}
\hline & $X X$ & $X Y$ & $X Z$ & $Y Y$ & $Y Z$ & $Z Z$ \\
\hline \multicolumn{7}{|l|}{$\mathrm{O}(1)$} \\
\hline PBE/6-31G** & -0.43 & -1.52 & 0.04 & 1.44 & 0.02 & -1.01 \\
\hline PBE/aug-cc-pVDZ & -0.41 & -1.50 & 0.03 & 1.37 & 0.02 & -0.96 \\
\hline PBE/aug-cc-pVDZ & -0.34 & -1.49 & 0.03 & 1.44 & 0.02 & -1.09 \\
\hline $\mathrm{XD} / \mathrm{PBE} / 6-31 \mathrm{G}^{* *}$ & -0.27 & -1.21 & -0.04 & 1.25 & 0.07 & -0.98 \\
\hline \multicolumn{7}{|l|}{$\mathrm{N}(2)$} \\
\hline $\mathrm{PBE} / 6-31 \mathrm{G}^{* *}$ & -0.61 & -0.03 & -0.05 & -0.66 & -0.01 & 1.27 \\
\hline PBE/aug-cc-pVDZ & -0.58 & -0.04 & -0.05 & -0.63 & -0.01 & 1.20 \\
\hline PBE/aug-cc-pVDZ & -0.56 & -0.03 & -0.05 & -0.59 & -0.01 & 1.15 \\
\hline $\mathrm{XD} / \mathrm{PBE} / 6-31 \mathrm{G}^{* *}$ & -0.39 & -0.02 & -0.02 & -0.45 & -0.02 & 0.85 \\
\hline \multicolumn{7}{|l|}{$\mathrm{C}(3)$} \\
\hline $\mathrm{PBE} / 6-31 \mathrm{G}^{* *}$ & 0.13 & 0.08 & 0.00 & 0.33 & 0.00 & -0.46 \\
\hline PBE/aug-cc-pVDZ & 0.16 & 0.08 & 0.00 & 0.34 & 0.00 & -0.49 \\
\hline PBE/aug-cc-pVDZ & 0.19 & 0.08 & 0.00 & 0.39 & 0.00 & -0.59 \\
\hline $\mathrm{XD} / \mathrm{PBE} / 6-31 \mathrm{G}^{* *}$ & 0.25 & 0.07 & 0.00 & 0.27 & -0.02 & -0.52 \\
\hline \multicolumn{7}{|l|}{$\mathrm{H}(4)$} \\
\hline $\mathrm{PBE} / 6-31 \mathrm{G}^{* *}$ & -0.32 & -0.42 & -0.02 & -0.06 & -0.01 & 0.37 \\
\hline PBE/aug-cc-pVDZ & -0.31 & -0.43 & -0.02 & -0.05 & -0.01 & 0.37 \\
\hline PBE/aug-cc-pVDZ & -0.31 & -0.42 & -0.02 & -0.05 & -0.01 & 0.36 \\
\hline $\mathrm{XD} / \mathrm{PBE} / 6-31 \mathrm{G} * *$ & -0.33 & -0.44 & -0.02 & -0.04 & -0.01 & 0.37 \\
\hline \multicolumn{7}{|l|}{$\mathrm{H}(5)$} \\
\hline $\mathrm{PBE} / 6-31 \mathrm{G}^{* *}$ & 0.25 & 0.07 & 0.00 & -0.60 & 0.00 & 0.35 \\
\hline PBE/aug-cc-pVDZ & 0.26 & 0.07 & 0.00 & -0.60 & 0.00 & 0.35 \\
\hline PBE/aug-cc-pVDZ & 0.25 & 0.07 & 0.00 & -0.60 & 0.00 & 0.34 \\
\hline $\mathrm{XD} / \mathrm{PBE} / 6-31 \mathrm{G} * *$ & 0.27 & 0.07 & 0.00 & -0.62 & 0.00 & 0.35 \\
\hline \multicolumn{7}{|l|}{$\mathrm{H}(6)$} \\
\hline $\mathrm{PBE} / 6-31 \mathrm{G}^{* *}$ & 0.21 & 0.01 & 0.00 & -0.44 & -0.01 & 0.23 \\
\hline PBE/aug-cc-pVDZ & 0.22 & 0.02 & 0.00 & -0.47 & -0.01 & 0.24 \\
\hline PBE/aug-cc-pVDZ & 0.20 & 0.01 & 0.00 & -0.43 & -0.01 & 0.23 \\
\hline $\mathrm{XD} / \mathrm{PBE} / 6-31 \mathrm{G}^{* *}$ & 0.21 & 0.01 & 0.00 & -0.46 & 0.00 & 0.24 \\
\hline
\end{tabular}

Nevertheless, the methods were published and are thus in the public domain and subject to further discussion.

The 1957 statement that 'only the traceless EFG tensor is relevant in the computation of quantities such as nuclear quadrupole coupling constants measured in NMR or NQR experiments (Cohen \& Reif, 1957)' is the basis for criticism of our comparison of the elements of the unabridged tensor elements. The point was to verify that our expressions yield correct values, for which the unabridged tensor provides a more stringent test. Going from the total to the traceless definition is of course trivial. In fact XD2006, like Gaussian 03 (2004), automatically prints out both total and traceless components of the EFG tensor as well as the corresponding eigenvalues (Appendix $A$, Supplementary Material). ${ }^{\mathbf{1}}$ For completeness, the components of the traceless EFG tensor and its eigenvalues for formamide are given in Tables 4 and 5. They in no way contradict any of the statements presented in our original paper.

We agree that use of the Sternheimer correction (Sternheimer, 1986) is questionable and should be re-examined. However, any disagreement between our value and the most precise up-to-date determination of $Q\left({ }^{57} \mathrm{Fe}^{m}\right)$ (Dufek et al., 1995) is unlikely to be due to the use of the correction, which adds only $0.01 \times 10^{-28} \mathrm{~m}^{2}$ to the uncorrected value of $Q\left({ }^{57} \mathrm{Fe}^{m}\right)=0.11 \times 10^{-28} \mathrm{~m}^{2}$ (less than $10 \%$ ). Any disagreement is more likely to be due to experimental errors in the data, which are larger than the Sternheimer correction, as shown in our paper. Of course, this does not eliminate the need for more

\footnotetext{
${ }^{1}$ Supplementary material for this paper is available from the IUCr electronic archives (Reference: SH0187). Services for accessing this material are described at the back of the journal.
}

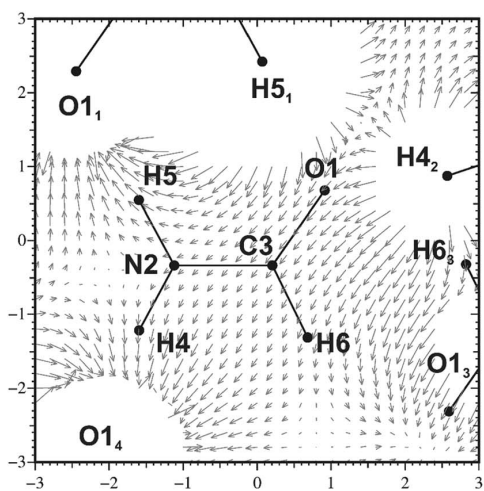

(a)

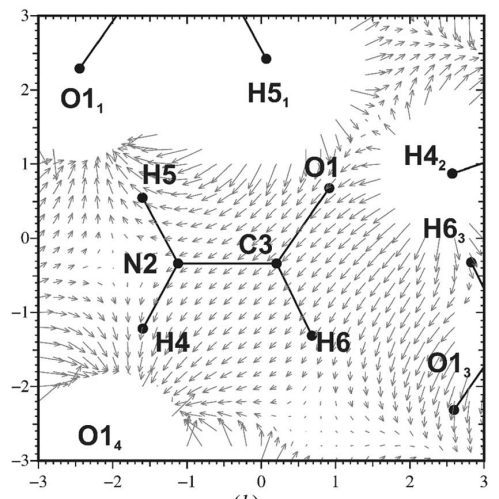

(b)
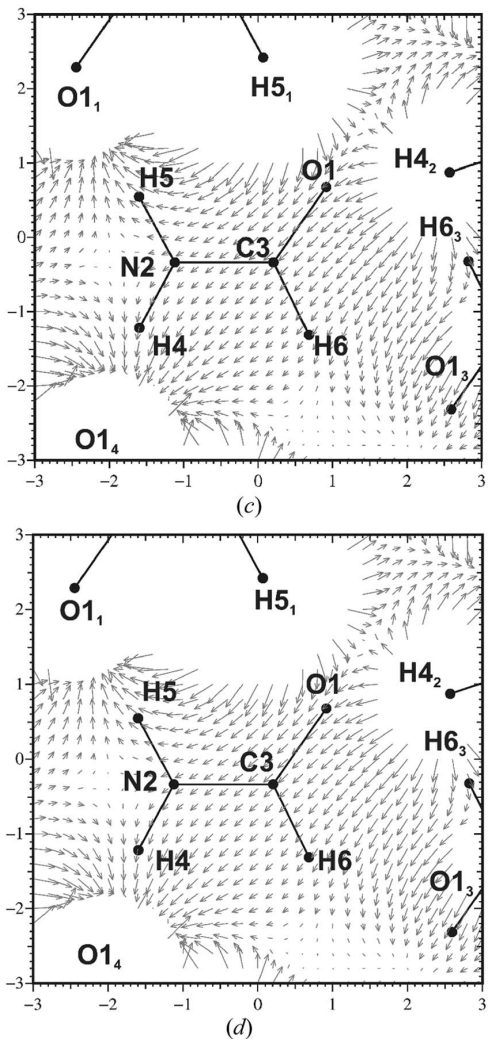

Figure 1

Electric field (EF) vectors in the $\mathrm{N} 2-\mathrm{C} 3-\mathrm{O} 1$ plane of the 'central' formamide molecule due to $(a)$ the eight nearest-neighbouring molecules in the crystal, and all atoms with fractional coordinates $(b)-1<x, y, z<2,(c)-2<x, y, z<3$ and (d) $-4<x, y, z<5$ (the contribution of the 'central' molecule to EF is not included). Subscripts of atom names identify the neighbouring molecules. Vectors with magnitudes larger than $0.15 \mathrm{e}^{-2}$ are omitted for clarity. The size of the map is $6 \times 6 \AA$ with a grid spacing of $0.2 \AA$. 
Table 5

Eigenvalues of the traceless EFG tensor at the nuclear positions in formamide (atomic units) from different methods.

\begin{tabular}{|c|c|c|c|}
\hline & $\lambda_{1}$ & $\lambda_{2}$ & $\lambda_{3}$ \\
\hline \multicolumn{4}{|l|}{$\mathrm{O}(1)$} \\
\hline $\mathrm{PBE} / 6-31 \mathrm{G} * *$ & -1.29 & -1.00 & 2.29 \\
\hline PBE/aug-cc-pVDZ & -1.27 & -0.95 & 2.22 \\
\hline PBE/aug-cc-pVDZ & -1.20 & -1.08 & 2.28 \\
\hline $\mathrm{XD} / \mathrm{PBE} / 6-31 \mathrm{G}^{* *}$ & -0.98 & -0.94 & 1.92 \\
\hline \multicolumn{4}{|l|}{$\mathrm{N}(2)$} \\
\hline $\mathrm{PBE} / 6-31 \mathrm{G}^{* *}$ & -0.68 & -0.59 & 1.27 \\
\hline PBE/aug-cc-pVDZ & -0.65 & -0.56 & 1.20 \\
\hline PBE/aug-cc-pVDZ & -0.61 & -0.54 & 1.15 \\
\hline $\mathrm{XD} / \mathrm{PBE} / 6-31 \mathrm{G}^{* *}$ & -0.46 & -0.39 & 0.85 \\
\hline \multicolumn{4}{|l|}{$\mathrm{C}(3)$} \\
\hline $\mathrm{PBE} / 6-31 \mathrm{G} * *$ & -0.46 & 0.10 & 0.35 \\
\hline PBE/aug-cc-pVDZ & -0.49 & 0.13 & 0.37 \\
\hline PBE/aug-cc-pVDZ & -0.59 & 0.16 & 0.42 \\
\hline $\mathrm{XD} / \mathrm{PBE} / 6-31 \mathrm{G}^{* *}$ & -0.52 & 0.19 & 0.33 \\
\hline \multicolumn{4}{|l|}{$\mathrm{H}(4)$} \\
\hline $\mathrm{PBE} / 6-31 \mathrm{G}^{* *}$ & -0.63 & 0.26 & 0.37 \\
\hline PBE/aug-cc-pVDZ & -0.63 & 0.26 & 0.37 \\
\hline PBE/aug-cc-pVDZ & -0.63 & 0.26 & 0.37 \\
\hline $\mathrm{XD} / \mathrm{PBE} / 6-31 \mathrm{G}^{* *}$ & -0.65 & 0.28 & 0.37 \\
\hline \multicolumn{4}{|l|}{$\mathrm{H}(5)$} \\
\hline $\mathrm{PBE} / 6-31 \mathrm{G} * *$ & -0.61 & 0.25 & 0.35 \\
\hline PBE/aug-cc-pVDZ & -0.61 & 0.26 & 0.35 \\
\hline PBE/aug-cc-pVDZ & -0.60 & 0.26 & 0.35 \\
\hline $\mathrm{XD} / \mathrm{PBE} / 6-31 \mathrm{G} * *$ & -0.62 & 0.28 & 0.35 \\
\hline \multicolumn{4}{|l|}{$\mathrm{H}(6)$} \\
\hline $\mathrm{PBE} / 6-31 \mathrm{G} * *$ & -0.44 & 0.21 & 0.23 \\
\hline PBE/aug-cc-pVDZ & -0.47 & 0.22 & 0.24 \\
\hline PBE/aug-cc-pVDZ & -0.43 & 0.20 & 0.23 \\
\hline $\mathrm{XD} / \mathrm{PBE} / 6-31 \mathrm{G}^{* *}$ & -0.46 & 0.21 & 0.24 \\
\hline
\end{tabular}

accurate experimentation and re-examination of the importance of the core polarization.

Financial support of this work by the National Science Foundation (CHE0236317) is gratefully acknowledged.

\section{References}

Abramov, Yu. A., Volkov, A. \& Coppens, P. (2000). J. Mol. Struct. (Theochem.), 529, 27-35.

Abramov, Yu. A., Volkov, A., Wu, G. \& Coppens, P. (2000a). Acta Cryst. A56, 585-591.

Abramov, Yu. A., Volkov, A., Wu, G. \& Coppens, P. (2000b). J. Phys. Chem. B104, 2183-2188.

Brown, A. S. \& Spackman, M. A. (1994). Mol. Phys. 83, 551-566.

Cohen, M. H. \& Reif, F. (1957). Solid State Phys. Adv. Res. Applic. 5, 321-438.

Cummins, P. G., Dunmur, D. A., Munn, R. W. \& Newham, R. J. (1976). Acta Cryst. A32, 847-853.

Dufek, P., Blaha, P. \& Schwarz, K. (1995). Phys. Rev. Lett. 25, 3545-3548.

Gaussian 03 (2004). Revision C.02, by M. J. Frisch et al. Gaussian, Inc., Wallingford, CT, USA.

Sternheimer, R. M. (1986). Z. Naturforsch. Teil A, 41, 24-36.

Su, Z. \& Coppens, P. (1995). Acta Cryst. A51, 27-32.

Volkov, A., King, H. F., Coppens, P. \& Farrugia, L. J. (2006). Acta Cryst. A62, $400-408$.

Volkov, A., King, H. F., Coppens, P. \& Macchi, P. (2007). In preparation.

Volkov, A., Koritsanszky T. S. \& Coppens, P. (2004). Chem. Phys. Lett. 391, 170-175.

Volkov, A., Macchi, P., Farrugia, L. J., Gatti, C., Mallinson, P., Richter, T. \& Koritsanszky, T. (2006). XD2006 - A Computer Program Package for Multipole Refinement, Topological Analysis of Charge Densities and Evaluation of Intermolecular Energies from Experimental or Theoretical Structure Factors. University at Buffalo, State University of New York, NY, USA; University of Milano, Italy; University of Glasgow, UK; CNRISTM, Milano, Italy; Middle Tennessee State University, TN, USA. 